\title{
Update on the Pediatric Dermatology Workforce Shortage
}

\author{
Teresa S. Wright, MD
}

$\square$ ediatric dermatology is a relatively young subspecialty. The Society for Pediatric Dermatology (SPD) was established in 1975, followed by the creation of the journal Pediatric Dermatology in 1982 and the American Academy of Pediatrics Section on Dermatology in $1986 .{ }^{1}$ In 2000, the Accreditation Council for Graduate Medical Education (ACGME) officially recognized pediatric dermatology as a unique subspecialty of the American Board of Dermatology (ABD). During that time, informal fellowship experiences emerged, and formal 1-year training programs approved by the ABD evolved by 2006. A subspecialty certification examination was created and has been administered every other year since $2004 .{ }^{1}$ Data provided by the SPD indicate that approximately 431 US dermatologists have passed the ABD's pediatric dermatology board certification examination thus far (unpublished data, September 2021).

In 1986, the first systematic evaluation of the US pediatric dermatology workforce revealed a total of 57 practicing pediatric dermatologists and concluded that job opportunities appeared to be limited at that time. ${ }^{2}$ Since then, the demand for pediatric dermatology services has continued to grow steadily, and the number of board-certified pediatric dermatologists practicing in the United States has increased to at least 317 per data from a 2020 survey. $^{3}$ However, given that there are more than 11,000 boardcertified dermatologists in the United States, there continues to be a severe shortage of pediatric dermatologists. ${ }^{1}$

\section{Increased Demand for Pediatric Dermatologists}

Approximately $10 \%$ to $30 \%$ of almost 200 million annual outpatient pediatric primary care visits involve a skin concern. Although many of these problems can be handled by primary care physicians, more than $80 \%$ of pediatricians report having difficulty accessing dermatology services for their patients. ${ }^{4}$ In surveys of pediatricians, pediatric dermatology has the third highest referral rate but has consistently ranked third among the specialties deemed most difficult to access. ${ }^{5-7}$ In addition, it is not uncommon for the wait time to see a pediatric dermatologist to be 6 weeks or longer. . $^{5}$

Recent population data estimate that there are 73 million children living in the United States. ${ }^{9}$ If there are roughly 317 practicing board-certified pediatric dermatologists, that translates into approximately 4.3 pediatric dermatologists per million children. This number is far smaller than the 4 general dermatologists per 100,000 individuals recommended by Glazer et $\mathrm{al}^{10}$ in 2017. To meet this suggested ratio goal, the workforce of pediatric dermatologists would have to increase to 2920. In addition to this severe workforce shortage, there is an additional problem with geographic maldistribution of pediatric dermatologists. More than $98 \%$ of pediatric dermatologists practice in metropolitan areas. At least 8 states and $95 \%$ of counties have no pediatric dermatologist, and there are no pediatric dermatologists practicing in rural counties. ${ }^{9}$ This disparity has considerable implications for barriers to care and lack of access for children living in underserved areas. Suggestions for attracting pediatric dermatologists to practice in these areas have included loan forgiveness programs as well as remote mentorship programs to provide professional support..$^{8,9}$

\section{Training in Pediatrics}

There currently are 38 ABD-approved pediatric dermatology fellowship training programs in the United States. Beginning in 2009, pediatric dermatology fellowship programs have participated in the SF Match program. Data provided by the SPD show that, since 2012, up to 27 programs have participated in the annual Match, offering a total number of positions ranging from 27 to 38; however, only 11 to 21 positions have been filled each year, leaving a large number of post-Match vacancies (unpublished data, September 2021).

Surveys have explored the reasons behind this lack of interest in pediatric dermatology training among

From Le Bonheur Children's Hospital and The University of Tennessee Health Science Center, Memphis.

The author reports no conflict of interest.

Correspondence: Teresa S. Wright, MD, LeBonheur Children's Hospital, Faculty Office Bldg, 49 N Dunlap St, Rm 102 , Memphis, TN 38105 (twrigh43@uthsc.edu).

doi:10.12788/cutis.0379 
dermatology residents. Factors that have been mentioned include lack of exposure and mentorship in medical school and residency, the financial hardship of an additional year of fellowship training, and historically lower salaries for pediatric dermatologists compared to general dermatologists. ${ }^{3,6}$

A 2004 survey revealed that more than $75 \%$ of dermatology department chairs believed it was important to have a pediatric dermatologist on the faculty; however, at that time only $48 \%$ of dermatology programs reported having at least 1 full-time pediatric dermatology faculty member. ${ }^{11}$ By 2008, a follow-up survey showed an increase to $70 \%$ of dermatology training programs reporting at least 1 full-time pediatric dermatologist; however, $43 \%$ of departments still had at least 1 open position, and $76 \%$ of those programs shared that they had been searching for more than 1 year. ${ }^{2}$ Currently, the Accreditation Data System of the ACGME shows a total of 144 accredited US dermatology training programs. Of those, 117 programs have 1 or more board-certified pediatric dermatology faculty member, and 27 programs still have none (unpublished data, September 2021).

A shortage of pediatric dermatologists in training programs contributes to the lack of exposure and mentorship for medical students and residents during a critical time in professional development. Studies show that up to $91 \%$ of pediatric dermatologists decided to pursue training in pediatric dermatology during medical school, pediatrics residency, or dermatology residency. In one survey, $84 \%$ of respondents $(\mathrm{N}=109)$ cited early mentorship as the most important factor in their decision to pursue pediatric dermatology. 6

A lack of pediatric dermatologists also results in suboptimal dermatology training for residents who care for children in primary care specialties, including pediatrics, combined internal medicine and pediatrics, and family practice. Multiple surveys have shown that many pediatricians feel they received inadequate training in dermatology during residency. Up to $38 \%$ have cited a need for more pediatric dermatology education $(\mathrm{N}=755) .5,6$ In addition, studies show a wide disparity in diagnostic accuracy between dermatologists and pediatricians, with one concluding that more than one-third of referrals to pediatric dermatologists were initially misdiagnosed and/or incorrectly treated. 5,7

\section{Recruitment Efforts for Pediatric Dermatologists}

There are multiple strategies for recruiting trainees into the pediatric dermatology workforce. First, given the importance of early exposure to the field and role models/ mentors, pediatric dermatologists must take advantage of every opportunity to interact with medical students and residents. They can share their genuine enthusiasm and love for the specialty while encouraging and supporting those who show interest. They also should seek opportunities for teaching, lecturing, and advising at every level of training. In addition, they can enhance visibility of the specialty by participating in career forums and/or assuming leadership roles within their departments or institutions. ${ }^{12}$ Another suggestion is for dermatology training programs to consider giving priority to qualified applicants who express sincere interest in pursuing pediatric dermatology training (including those who have already completed pediatrics residency). Although a 2008 survey revealed that $39 \%$ of dermatology residency programs $(\mathrm{N}=80)$ favored giving priority to applicants demonstrating interest in pediatric dermatology, others were against it, citing issues such as lack of funding for additional residency training, lack of pediatric dermatology mentors within the program, and an overall mistrust of applicants' sincerity. ${ }^{2}$

\section{Final Thoughts}

The subspecialty of pediatric dermatology has experienced remarkable growth over the last 40 years; however, demand for pediatric dermatology services has continued to outpace supply, resulting in a persistent and notable workforce shortage. Overall, the current supply of pediatric dermatologists can neither meet the clinical demands of the pediatric population nor fulfill academic needs of existing training programs. We must continue to develop novel strategies for increasing the pool of students and residents who are interested in pursuing careers in pediatric dermatology. Ultimately, we also must create incentives and develop tactics to address the geographic maldistribution that exists within the specialty.

\section{REFERENCES}

1. Prindaville B, Antaya R, Siegfried E. Pediatric dermatology: past, present, and future. Pediatr Dermatol. 2015;32:1-12.

2. Craiglow BG, Resneck JS, Lucky AW, et al. Pediatric dermatology workforce shortage: perspectives from academia. J Am Acad Dermatol. 2008;59:986-989.

3. Ashrafzadeh S, Peters G, Brandling-Bennett H, et al. The geographic distribution of the US pediatric dermatologist workforce: a national cross-sectional study. Pediatr Dermatol. 2020;37:1098-1105.

4. Stephens MR, Murthy AS, McMahon PJ. Wait times, health care touchpoints, and nonattendance in an academic pediatric dermatology clinic. Pediatr Dermatol. 2019;36:893-897.

5. Prindaville B, Simon S, Horii K. Dermatology-related outpatient visits by children: implications for workforce and pediatric education. J Am Acad Dermatol. 2016;75:228-229.

6. Admani S, Caufield M, Kim S, et al. Understanding the pediatric dermatology workforce shortage: mentoring matters. I Pediatr. 2014;164:372-375.

7. Fogel AL, Teng JM. The US pediatric dermatology workforce: an assessment of productivity and practice patterns. Pediatr Dermatol. 2015;32:825-829.

8. Prindaville B, Horii K, Siegfried E, et al. Pediatric dermatology workforce in the United States. Pediatr Dermatol. 2019;36:166-168.

9. Ugwu-Dike P, Nambudiri V. Access as equity: addressing the distribution of the pediatric dermatology workforce [published online August 2, 2021]. Pediatr Dermatol. doi:10.1111/pde.14665

10. Glazer AM, Rigel DS. Analysis of trends in geographic distribution of US dermatology workforce density. JAMA Dermatol. 2017; 153:472-473.

11. Hester EJ, McNealy KM, Kelloff JN, et al. Demand outstrips supply of US pediatric dermatologists: results from a national survey. J Am Acad Dermatol. 2004;50:431-434.

12. Wright TS, Huang JT. Comment on" pediatric dermatology workforce in the United States". Pediatr Dermatol. 2019;36:177-178. 